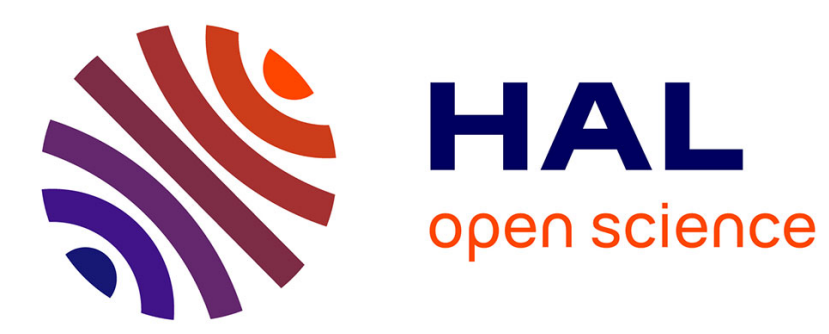

\title{
The effect of toroidicity on Reversed Field Pinch dynamics
}

Jorge A. Morales, Wouter J.T. Bos, Kai Schneider, David Montgomery

\section{To cite this version:}

Jorge A. Morales, Wouter J.T. Bos, Kai Schneider, David Montgomery. The effect of toroidicity on Reversed Field Pinch dynamics. Plasma Physics and Controlled Fusion, 2014, 56, pp.095024 10.1088/0741-3335/56/9/095024 . hal-01026354

\section{HAL Id: hal-01026354 https://hal.science/hal-01026354}

Submitted on 31 Jul 2014

HAL is a multi-disciplinary open access archive for the deposit and dissemination of scientific research documents, whether they are published or not. The documents may come from teaching and research institutions in France or abroad, or from public or private research centers.
L'archive ouverte pluridisciplinaire HAL, est destinée au dépôt et à la diffusion de documents scientifiques de niveau recherche, publiés ou non, émanant des établissements d'enseignement et de recherche français ou étrangers, des laboratoires publics ou privés. 


\title{
On the effect of Toroidicity on Reversed Field Pinch Dynamics
}

\author{
Jorge A. Morales \\ LMFA, CNRS, École Centrale de Lyon, France
}

Wouter J.T. Bos

LMFA, CNRS, École Centrale de Lyon, France

\section{Kai Schneider}

M2P2, CMI, CNRS, Aix-Marseille Université, France

\author{
David C. Montgomery \\ Department of Physics and Astronomy, Dartmouth College, NH, USA
}

\begin{abstract}
The influence of the curvature of the imposed magnetic field on Reversed Field Pinch dynamics is investigated by comparing the flow of a magnetofluid in a torus with aspect ratio 1.83, with the flow in a periodic cylinder. It is found that an axisymmetric toroidal mode is always present in the toroidal, but absent in the cylindrical configuration. In particular, in contrast to the cylinder, the toroidal case presents a double poloidal recirculation cell with a shear localized at the plasma edge. Quasi-single-helicity states are found to be more persistent in toroidal than in periodic cylinder geometry.
\end{abstract}

PACS numbers: 52.55.Lf, $52.65 . \mathrm{Kj}, 52.30 . \mathrm{Cv}$ 


\section{Introduction}

For the last decades research on magnetically controlled fusion has mainly focused on toroidal devices such as the tokamak, stellarator or Reversed Field Pinch (RFP). Of these devices the tokamak has received most interest due to its geometrical simplicity as compared to the stellarator and the stability of its magnetic field as compared to the RFP. This latter device possesses the technical advantage that the imposed toroidal magnetic field needs not to be as large as for a tokamak, since the self-organized state enhances the magnetic field through a mechanism akin to the dynamo effect often invoked to explain the origin of the magnetic field of the Earth. This allows for the use of less sophisticated coils to generate the toroidal magnetic field. However, it was shown that RFP devices are plagued by magnetohydrodynamic (MHD) instabilities, leading to a self-organized turbulent state which degrades the confinement quality. This chaotic regime is called a multiple-helicity $(\mathrm{MH})$ state because a multitude of helically extended modes interact. The term helicity is used here to indicate the shape of the modes, which have a poloidal and toroidal spatial frequency both different from zero.

However, in the last two decades quasi-single helicity (QSH) states were observed in RFP experiments, where the full turbulent regime is avoided and the energy of one helical mode predominates above the others $[1,2,3,4,5]$. In the QSH state there is a decrease of magnetic chaos and the formation of a coherent helical structure within the plasma. The reduction of particle transport was observed in QSH states with respect to $\mathrm{MH}$ states $[6,7,8]$. Also it was found that increasing the toroidal current makes the QSH regime more persistent $[9,10]$. The QSH state is responsible for the creation of internal transport barriers (ITB) that improve the confinement time by a factor of two $[11,12]$. Recently it was discovered that external transport barriers (ETB) are created for low plasma densities that increase by $30 \%$ the confinement time. The origin of these barriers is not well understood but it could be related to shear flows [13]. Given the practical advantages of the RFP design, the discovery of this QSH state renewed the interest of the fusion community in the RFP dynamics.

The investigation of a cylinder, periodic in the axial direction, is analytically more tractable than a torus since a cylinder contains translational invariance both in axial and azimuthal direction, whereas in a torus only translational invariance exists in the toroidal direction. In the case in which the ratio of the larger to smaller radius of a toroidal plasma tends to infinity, the effects of curvature become negligible and the plasma can be locally approximated by a straight cylinder. In practice this ratio is however in general not much larger than four so that such an approximation is, at least, questionable. Nevertheless, a certain number of important features are observed both in cylindrical and toroidal geometry. Due to the conceptual and technical simplicity of the periodic cylinder, the majority of numerical studies of the RFP, using the MHD approximation, consider therefore periodic cylindrical geometries, thereby ignoring the influence of the curvature of the magnetic field.

Numerical MHD simulations became important tools in the investigation of RFPs 
in the 1980s, e.g. $[14,15]$. In the 1990s an important body of results was produced using efficient codes in cylindrical geometry [16, 17]. In particular the role of the Hartmann number and pinch ratio on the triggering of helical modes were assessed in some detail in cylindrical geometry $[18,19,20]$, and more recently the role of compressibility [21, 22]. Recent studies in cylindrical geometry show some agreement with experiments $[23,24,25]$, but particularly important features such as the persistence of the QSH state remain to be elucidated. Indeed the QSH state seems to become more important in RFPs for increasing Lundquist number [12]. In simulations this has only been observed if a particular type of boundary conditions is imposed [25]. Ideal MHD equilibrium solvers allow to reproduce QSH equilibria [26], but need experimental input such as the position of the helical axis, pressure profile and safety-factor profile. Threedimensional codes solving the MHD equations in toroidal geometries without a priori definition of pressure and safety factor profiles exist, [27, 24, 28] but there is not yet, to our knowledge, a study which systematically investigates the influence of curvature on the dynamics of a toroidal magnetofluid in the RFP context. This motivates the present study which compares, using exactly the same numerical code and governing equations, the dynamics of a viscoresistive magnetofluid in a toroidal domain to those in a cylindrical domain. We can thereby disentangle the influence of the toroidicity from all other influences. By toroidicity we mean here the curvature of the magnetic field induced by the toroidal geometry. In particular will we keep the minor radius of the torus equal to the radius of the cylinder and will we fix the length of the cylinder to have the same value as the length of the toroidal axis. The toroidal domain corresponds then roughly to the cylindrical domain which has been bent to form a torus.

\section{Visco-resistive MHD simulations}

We consider non-ideal MHD in which both viscous and resistive effects are taken into account. An isothermal, incompressible plasma is considered with uniform and constant transport coefficients, which simplifies the problem as far as possible, while retaining the required level of complexity to study the emergence of helical modes in a self-consistent manner. Indeed, if we drop the resistive term and consider ideal MHD, the imposed toroidal electric field will become independent from the toroidal plasma current, an assumption which can dramatically change the plasma dynamics [29]. The Alfvénnormalized viscoresistive MHD equations are [30],

$$
\begin{aligned}
& \partial_{t} \boldsymbol{u}-M^{-1} \nabla^{2} \boldsymbol{u}=-\nabla p^{*}+\boldsymbol{u} \times \boldsymbol{\omega}+\boldsymbol{j} \times \boldsymbol{B}, \\
& \partial_{t} \boldsymbol{B}-S^{-1} \nabla^{2} \boldsymbol{B}=\nabla \times(\boldsymbol{u} \times \boldsymbol{B}),
\end{aligned}
$$

with $\nabla \cdot \boldsymbol{u}=0$ and $\nabla \cdot \boldsymbol{B}=0$, the current density $\boldsymbol{j}=\nabla \times \boldsymbol{B}$, the vorticity $\boldsymbol{\omega}=\nabla \times \boldsymbol{u}$ and the total pressure $p^{*}=p+u^{2} / 2$. These equations are non-dimensionalized using the poloidal Alfvén speed $C_{A}=B_{\text {ref }} / \sqrt{\rho \mu_{0}}$, with $B_{\text {ref }}$ the reference poloidal magnetic field, $\rho$ the density and $\mu_{0}$ the permeability of vacuum. Our choice of an isothermal incompressible description of the plasma is the simplest description which 
satistfies the conservation of mass. Two distinct geometries are considered: a torus and a periodic cylinder (see Fig. 1), both with circular cross-section. The reference length $L$ is the diameter of these cross sections. This normalization introduces two dimensionless quantities, $S=C_{A} L / \lambda$ and $M=C_{A} L / \nu$ the Lundquist and viscous Lundquist numbers, respectively, with $\lambda$ the magnetic diffusivity and $\nu$ the kinematic viscosity. The ratio of these two quantities is the magnetic Prandtl number $\operatorname{Pr}=\nu / \lambda$. We have chosen to consider spatially uniform viscosity and resistivity values. These assumptions should of course be refined if one wants to approach the behaviour of existing machines. We think, however, that it is not desirable to introduce this refinement in the present study, since it would impede us to disentangle the influence of the geometry on the dynamics from the influence induced by the spatial profile of the transport coefficients.

We fix the diameter $L \equiv 2 a=0.6 \pi$, and the axial length of the cylinder $2 \pi R_{0}$, where $R_{0}=0.55 \pi$ is the length from the axis to the center of the toroidal cross section, so that the cylinder has the same length as the toroidal axis passing through the center of the torus' poloidal cross-section. The torus has then an aspect ratio $\sim 1.83$. We have not carried out a systematic study on the influence of the aspect radius on the dynamics. Rather do we focus on the relatively compact torus compared to a straight cylinder to identify the influence of the presence of curvature of the magnetic field lines on the dynamics. A systematic study varying the aspect ratio would indeed constitute an interesting perspective. The reference poloidal magnetic field $B_{\text {ref }}=0.15 \pi$, is set such that the average toroidal or axial current-density are $\left\langle j_{T}\right\rangle=1$. For the toroidal geometry we impose a magnetic field $B_{0_{T}}(R) \propto 1 / R$. In a real device this field is produced by the toroidal field coils located at the exterior of the vessel. A toroidal electric field is also present, in order to induce a toroidal plasma current. This electric field is chosen $E_{0_{T}}(R) \propto 1 / R[31]$. For the present study we consider a constant conductivity, and therefore the induced toroidal current density field has the same dependence, $j_{0_{T}}(R) \propto 1 / R$. The toroidal magnetic and current density profiles give the imposed three-dimensional helical magnetic field $\boldsymbol{B}_{0}=\boldsymbol{B}_{0_{T}}+\boldsymbol{B}_{0_{p o l}}$. The poloidal magnetic field is calculated from the current density distribution $j_{0_{T}}(R)$. For more details about its numerical generation in general geometries we refer to [32]. For the cylindrical geometry the imposed axial magnetic and electric field are uniform. As the conductivity is considered uniform the induced current density field will be so as well. For a given geometry, the helical curvature of the magnetic field is in our system determined by a single parameter, the pinch ratio $\Theta$. Together with the value of the Prandtl number and the Lundquist number this parameter will determine the behaviour of the system. For the toroidal geometry $\Theta$ is defined as the wall averaged poloidal magnetic field over the volume averaged toroidal (or axial) magnetic field, $\Theta=\overline{B_{P}} /\left\langle B_{T}\right\rangle$.

The boundary conditions are no-slip, $\left.\boldsymbol{u}\right|_{\text {wall }}=0$, for the velocity. For the magnetic perturbations around the base field, the poloidal component and the component normal to the wall vanish, $B^{\prime}{ }_{P_{\text {wall }}}=B^{\prime}{ }_{\perp_{\text {wall }}}=0$, while the toroidal (or axial) component is free. The initial condition for the simulations are zero magnetic perturbations and zero velocity for the toroidal geometry. In the cylindrical case the magnetic perturbations are 

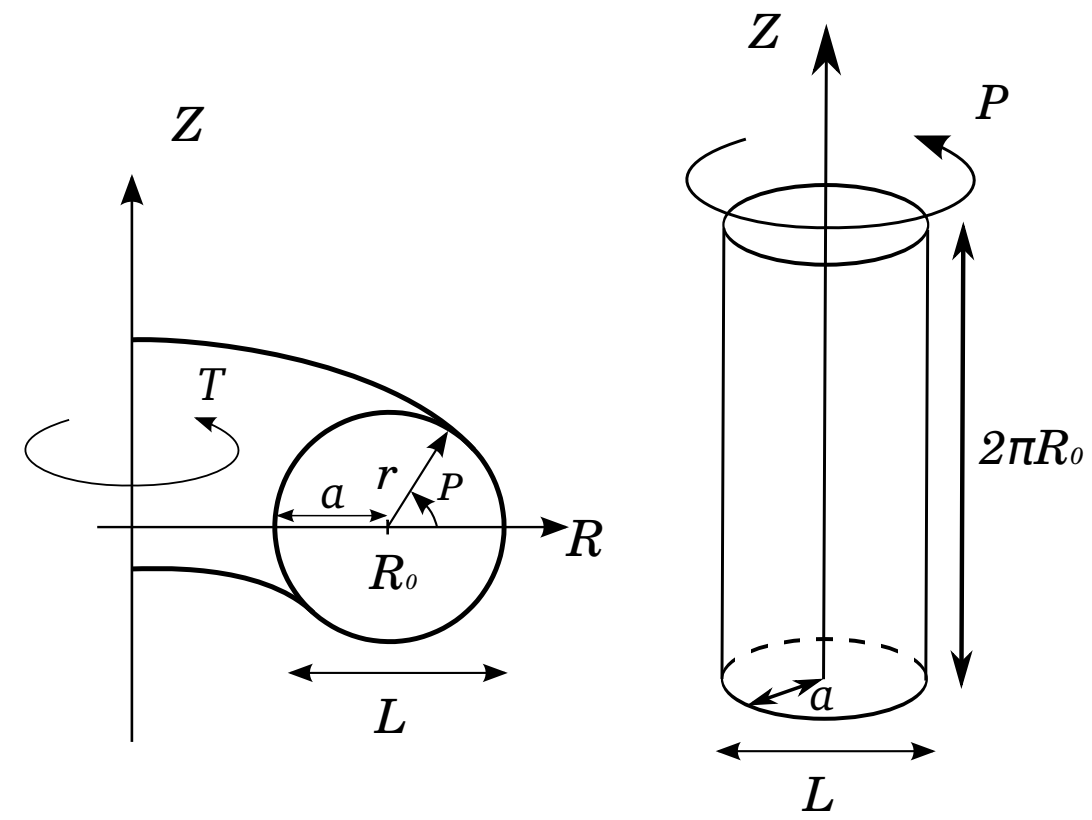

Figure 1. Toroidal (left) and periodic cylinder geometry (right). The toroidal direction is labeled $T$ and the poloidal $P$.

zero but a small white noise (with rms value of $10^{-4}$ ) is introduced in the initial velocity field in order to trigger the instability, a feature which is not necessary in the toroidal simulations. Equations $(1,2)$ are discretized with a Fourier pseudo-spectral method on a Cartesian grid. To impose the boundary conditions we use the volume-penalization technique. This method is benchmarked in detail for three-dimensional viscoresistive MHD equations in [33]. The simulations are carried out on a cubic domain with $128^{3}$ grid points for all simulations.

For the considered geometry, under our assumptions, the dynamics are entirely determined by the parameters $M, \operatorname{Pr}, \Theta$. In the present investigation we keep the magnetic Prandtl number fixed at $\operatorname{Pr}=3$ and we consider two different viscous Lundquist numbers $M=444$ and $M=888$. The value of the Prandtl number is chosen as a compromise between physical considerations and numerical efficiency. Indeed, $\operatorname{Pr}>1$ is expected to be found in fusion plasmas and was used in previous numerical studies $\operatorname{Pr} \in[1,20]$ [34]. However, high $\operatorname{Pr}$ number studies would limit the simulations to focus on highly viscous behaviour, which is less realistic. Since the value of the viscosity in fusion plasmas is not well defined, an investigation of the influence of the Prandtl number constitutes an interesting perspective. Here we shall focus in particular on the influence of the pinch ratio on the dynamics. All results are evaluated once the system has obtained a statistically steady state. 

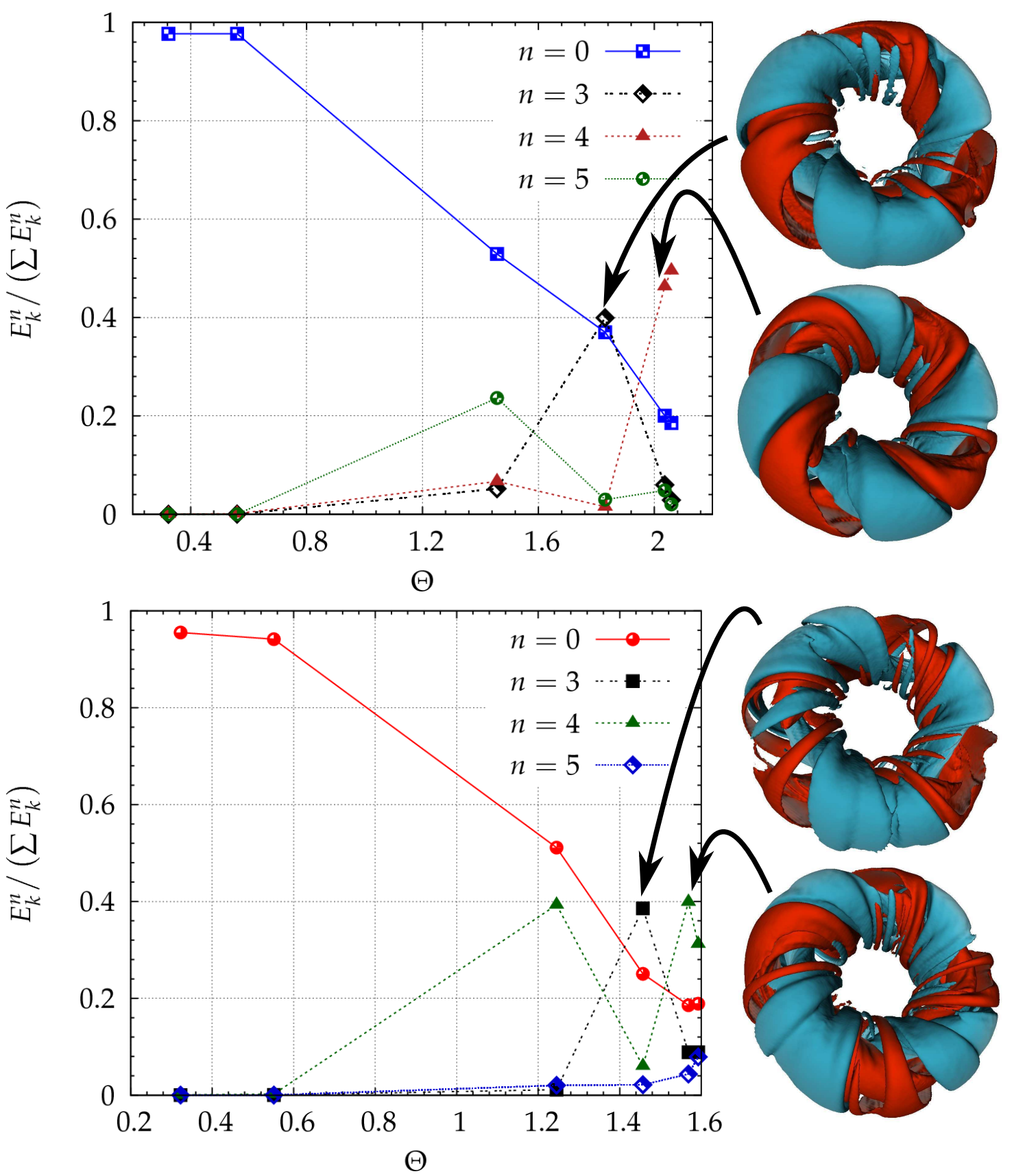

Figure 2. Ratio of the kinetic energy of the dominant toroidal modes over the total kinetic energy for the torus geometry, $M=444$ (top) and $M=888$ (bottom) as a function of $\Theta$. Visualization of the modes: toroidal velocity isosurfaces +0.007 (blue) and -0.007 (orange). 

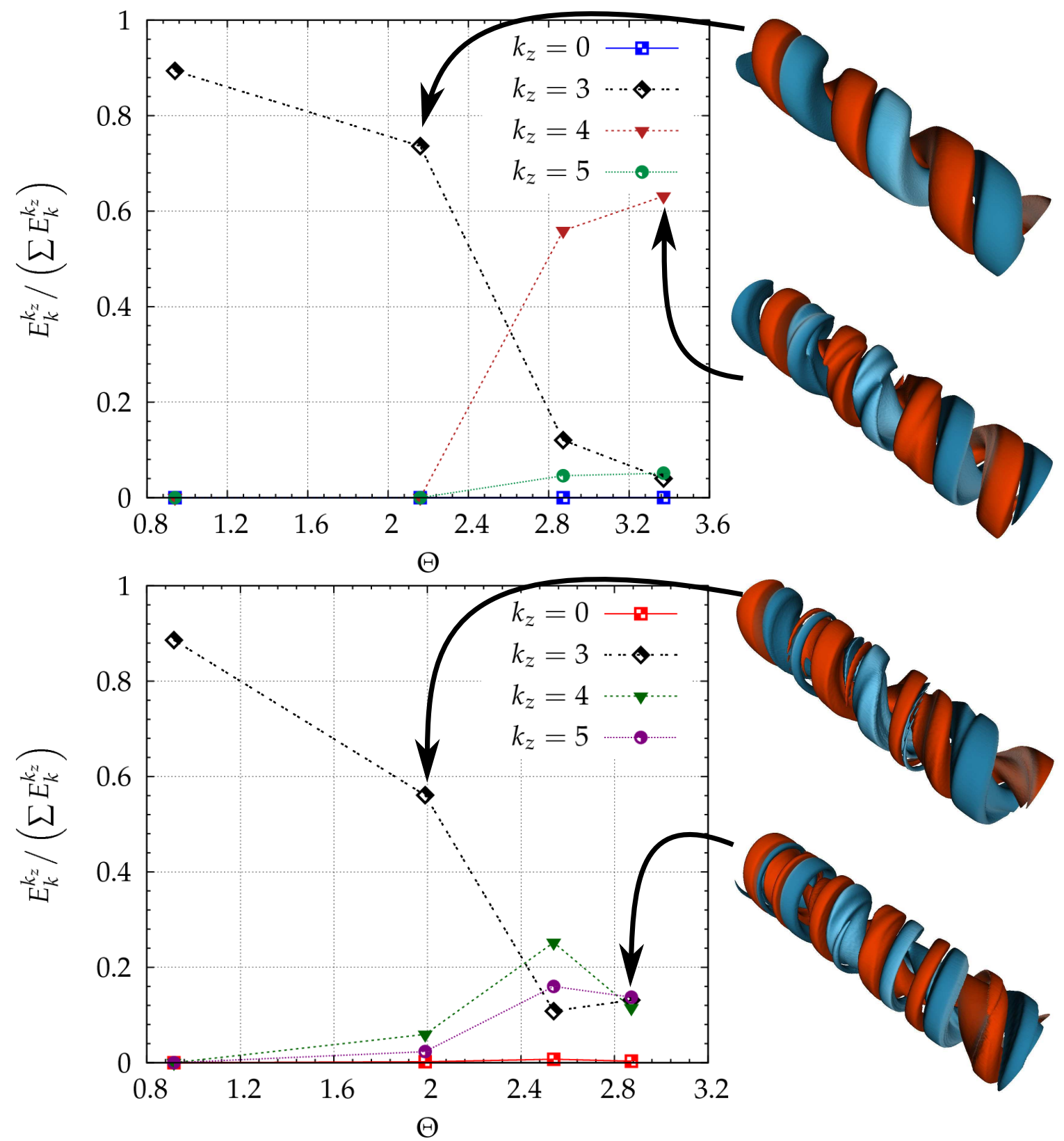

Figure 3. Ratio of the kinetic energy of the dominant axial modes over the total kinetic energy for the cylindrical geometry, $M=444$ (top) and $M=888$ (bottom) as a function of $\Theta$. Visualization of the modes: axial velocity isosurfaces +0.008 (blue) and -0.008 (orange).

\section{Results of the simulations}

In Fig. 2 it is shown that for a value of $\Theta<1$ the kinetic energy is mostly contained in the zero toroidal mode. This means that the velocity field for these parameters is axisymmetric around the major axis of the torus. At higher values of $\Theta$, roughly around $\Theta=1$, the helical modes with $n \neq 0$ become more important. But even at the highest values of $\Theta$ reported in the present investigation, the toroidal zero mode represents still 


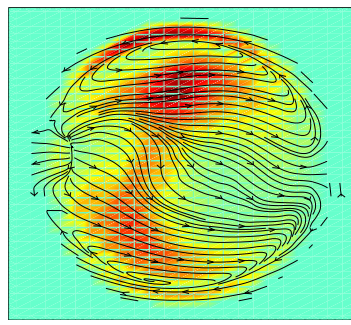

$(a)$

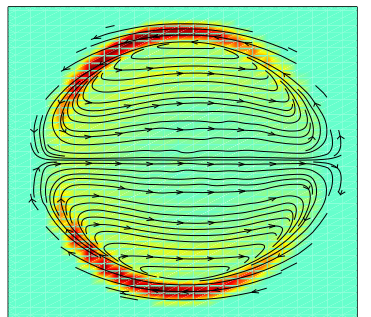

(b)

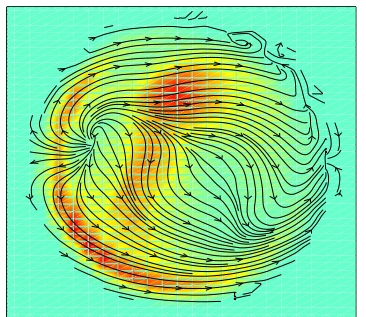

$(c)$

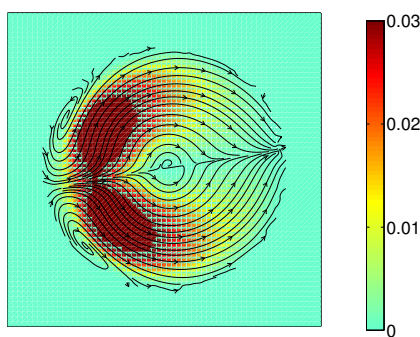

$(d)$

Figure 4. Poloidal velocity vector norm (color) and poloidal streamlines in the toroidal geometry (a-c) and cylinder (d). (a) - Total poloidal velocity field in a poloidal cut. (b) - The azimuthally averaged poloidal field (mode $n=0$ ). (c) - The total field $(a)$ minus the azimuthally averaged field $(b)$. For $M=444$ and $\Theta=1.83$. $(d)$ - Total poloidal velocity field in a poloidal cut in the cylinder for $M=444$ and $\Theta=2.16$.

$20 \%$ of the total kinetic energy and thus importantly affects the dynamics for all cases considered here. In contrast, in the cylindrical geometry the relative influence of the axial-invariant mode is negligible for all considered values of $M$ and $\Theta$. This marks an important difference: due to the curvature of the magnetic field, RFP dynamics will always be governed by a mix of helical modes, and toroidally invariant modes. These latter are absent in cylindrical geometry.

The dominant helical modes at the higher pinch ratios are $n$ or $k_{z}=3,4$ for the toroidal and cylindrical geometry (see Figs. 2 and 3). This result is in good agreement with experimental data from the RFP RELAX [35], which possesses an aspect ratio $\sim 2$ close to the one used in the present simulations. The dominant modes measured in this device are $n=4$ and $n=5$ [35]. For the simulations performed with $M=888$ there is an equipartition of the kinetic energy between more modes in the cylindrical geometry and the state is in a multiple-helicity state [1]. The toroidal simulations have a mode $n=4$ which continues to be significantly more energetic than the others. The toroidal geometry displays thus a state closer to a single-helicity state than the cylinder.

In Fig. $4(a)$, an instantaneous plot of the velocity field in a poloidal cross section is presented for the simulation with $M=444$ and $\Theta=1.83$ in the toroidal configuration. Fig. $4(b)$ shows the toroidally averaged poloidal flow, corresponding to the $n=0$ mode. This field is composed of two counter-rotating vortices, and is characterized by peaked poloidal velocities located in the external region where a shear zone exists. The kinetic energy of the zero mode is mainly localized at the plasma edge. The flow topology is similar in the torus at low pinch ratio $(\Theta<1)$. Such flow-fields were obtained analytically for large transport coefficients in [36] and numerically for relatively high Lundquist numbers in $[30,37]$. In more realistic tokamak-like configurations with Xpoint geometries using the MHD model with a bootstrap contribution a similar field was observed [38] as well as using the reduced MHD model [39]. Our results give some evidence that this axisymmetric field persists in the presence of the helical modes. In Fig. $4(c)$ we show the flow corresponding to the helical modes $n \neq 0$. The dominant mode is $n=3$. Hence this poloidal flow structure rotates three times around the 


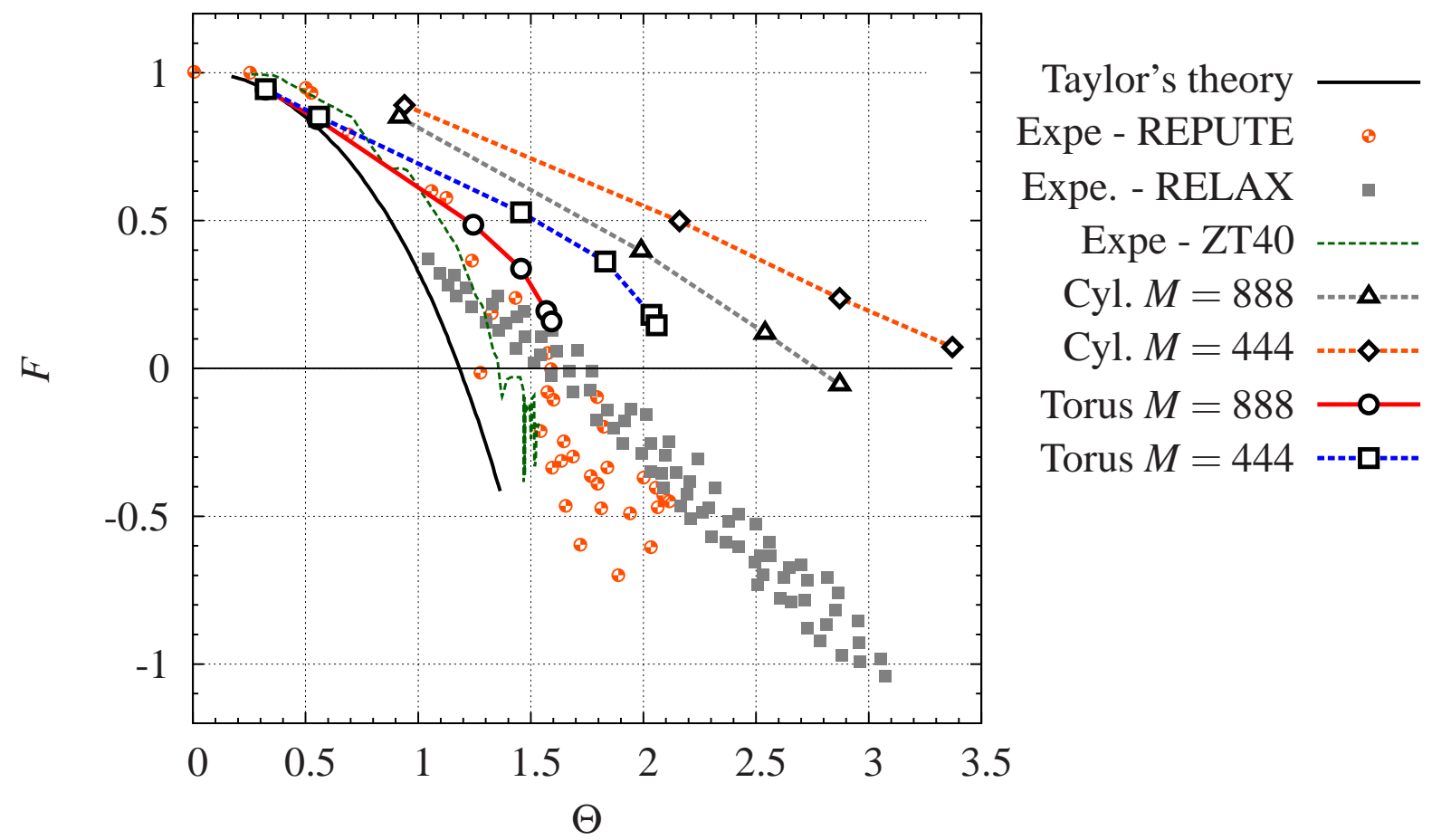

Figure 5. Field reversal parameter $F$ as a function of the pinch parameter $\Theta$ for the toroidal and cylindrical simulations and experimental data of three RFP devices.

toroidal axis for one toroidal loop. If the total poloidal flow is compared to the poloidal flow in the cylindrical geometry, Fig. $4(d)$, an important difference is observed. The double vortex flow pattern appearing in the torus is completely absent in the cylindrical geometry. This poloidal flow pattern is relevant because the steep gradients induced by these vortices can be responsible for the generation of internal transport barriers. A link between the presence of such internal transport barriers and the magnetic topology of the QSH state was recently suggested through an energy minimization argument [40].

\section{Comparison with experimental results}

The comparison of numerical simulations with experimental results is a rather complicated exercise in the field of fusion plasmas. The assumption of scalar and uniform transport coefficients is a bold approximation. Furthermore, the Lundquist numbers in realistic machines is orders of magnitude larger than what we have used in the present simulations. A detailed comparison with respect to local flow and magnetic field measurements seems thus not appropriate and we will therefore focus on two global parameters: the pinch ratio and the field-reversal parameter.

Quantitatively our numerical results are compared to experimental data of three different RFP devices. The first set of experimental data comes from the REPUTE experiment, described in [41, 42], the second set of data is the RFP ZT-40M [41, 43] and the third is from the device RELAX [35]. We recall that this last experiment has a low aspect ratio $\sim 2$, close to the aspect ratio used in the present simulations. This 
device has the particularity to allow $\Theta$ to be greater than two, whereas for the majority of RFP experiments the pinch ratio is around 1.5. In Fig. 5 our numerical and the experimental results are presented in the $\Theta-F$ plane. $F$ is the reversal parameter defined as the wall averaged toroidal magnetic field over the volume averaged toroidal magnetic field, $F=\overline{B_{T}} /\left\langle B_{T}\right\rangle$. We also show the theoretical curve found by Taylor [44] that has been recently critically reviewed in [45] with respect to its relevance to the RFP dynamics.

From Fig. 5 we can see that the simulations give results in the $\Theta-F$ plane comparable to those obtained in the RFP experiments: the reversal of the toroidal magnetic field $B_{T}$ for the considered experiments occurs around the same value, for $\Theta \approx 1.5$. The set of simulations that fits best the experiments is the one performed for a toroidal geometry with viscous Lundquist number $M=888$. In this figure we note that both the geometry and the Lundquist number play an important role in the evolution of the reversal parameter $F$ with $\Theta$. Using the toroidal geometry and increasing sufficiently the viscous Lundquist number, we fit better the experimental data. It is not yet clear whether we can get a magnetic surface averaged toroidal reversal at the torus edge for high enough values of $M$. In this study the computational resources where not sufficient to carry out higher resolution computations enabling higher viscous Lundquist and Prandtl number simulations. It seems that a reversed magnetic field is more easily obtained in the constant pressure description adopted in, for instance [34]. This explains, perhaps, that those results compare better to experiments. A drawback of such a MHD description is that it severely violates the conservation of mass of the system. We have therefore preferred to stay within the incompressible description of the plasma, which is the simplest description which allows to satisfy the mass-conservation principle. Further studies are needed to investigate to what extent one must take into account the conservation of mass, and compressibility effects in order to more realistically approach the behaviour of a RFP plasma.

In the present work, within the MHD description that we have adopted and in the parameter range that we have considered, the agreement of the $\Theta-F$ value with experiment seems to be closer for the toroidal than for the cylindrical RFP simulations.

\section{Conclusions}

The toroidal and cylindrical simulations carried out in the present study help to understand the role of toroidicity in the RFP dynamics. The helical instability appearing for increasing pinch ratio is quite similar for both cases. Generally, if the viscous Lundquist number is not too important a single helical mode dominates the system, so that we are close to the quasi-single helicity state found in experiments. For increasing viscous Lundquist number a turbulent state appears at large pinch ratio, showing equipartition of the energy between the helical modes in the cylindrical geometry, whereas, for the parameters considered in the present case, a single helical mode remains dominant in the toroidal geometry. It seems that the QSH state is more persistent in 
toroidal than in cylindrical configuration. Another fundamental qualitative difference between the cylinder and the torus is the axisymmetric flow that disappears in the cylindrical case. The toroidally invariant poloidal flow is composed of two counterrotating vortices. In a straight cylinder this characteristic of the plasma dynamics does not appear. Quantitatively, better agreement of the $F(\Theta)$ dependence is observed for toroidal simulations.

An important perspective is the study of the influence of more sophisticated resistivity profiles on the dynamics. This is currently under investigation. Since we have shown that the toroidicity influences the dynamics, a refined study of the influence of the aspect ratio on RFP dynamics would be a valuable contribution to the field. Further desirable refinements are the implementation of an equation of state and, of course, higher resolution simulations in order to reach more realistic values of the Lundquist and magnetic Prandtl number.

\section{Acknowledgments}

This work was supported by the contract SiCoMHD (ANR-Blanc 2011-045), computing time was supplied by IDRIS, project 22206 .

\section{References}

[1] D.F. Escande, P. Martin, S. Ortolani, A. Buffa, P. Franz, L. Marrelli, E. Martines, G. Spizzo, S. Cappello, A. Murari, R. Pasqualotto, and P. Zanca. Quasi-single-helicity reversed-field-pinch plasmas. Phys. Rev. Lett., 85(8):1662-5, 2000.

[2] P. Martin et al. Quasi-single helicity states in the reversed field pinch: Beyond the standard paradigm. Phys. Plasmas, 7(5):1984, 2000.

[3] P. Nordlund. The structure and stability of the reversed field pinch magnetic equilibrium in Extrap T1. Physica Scripta, 49(2):239, 1994.

[4] P.R. Brunsell, Y. Yagi, Y. Hirano, Y. Maejima, and T. Shimada. Coherent magnetic field fluctuations and locked modes in a reversed-field pinch. Phys. Fluids B, 5:885, 1993.

[5] J.S. Sarff, N.E. Lanier, S.C. Prager, and M.R. Stoneking. Increased confinement and $\beta$ by inductive poloidal current drive in the reversed field pinch. Phys. Rev. Lett., 78(1):62, 1997.

[6] L. Frassinetti, P.R. Brunsell, and J.R. Drake. Experiments and modelling of active quasi-single helicity regime generation in a reversed field pinch. Nucl. Fusion, 49(7):075019, 2009.

[7] I. Predebon, L. Marrelli, R.B. White, and P. Martin. Particle-transport analysis in reversed field pinch helical states. Phys. Rev. Lett., 93(14):145001, 2004.

[8] M. Gobbin, L. Marrelli, and R.B. White. Numerical studies of transport mechanisms in RFX-mod low magnetic chaos regimes. Plasma Phys. Controlled Fusion, 51(6):065010, 2009.

[9] P. Martin, L. Marrelli, A. Alfier, F. Bonomo, D.F. Escande, P. Franz, L. Frassinetti, M. Gobbin, R. Pasqualotto, P. Piovesan, et al. A new paradigm for RFP magnetic self-organization: results and challenges. Plasma Phys. Controlled Fusion, 49(5A):A177, 2007.

[10] R. Lorenzini, E. Martines, P. Piovesan, D. Terranova, P. Zanca, M. Zuin, A. Alfier, D. Bonfiglio, F. Bonomo, A. Canton, et al. Self-organized helical equilibria as a new paradigm for ohmically heated fusion plasmas. Nature Phys., 5(8):570-574, 2009.

[11] L. Carraro, A. Alfier, F. Bonomo, A. Fassina, M. Gobbin, R. Lorenzini, P. Piovesan, M.E. Puiatti, G. Spizzo, D. Terranova, et al. Improved confinement with internal electron transport barriers in RFX-mod. Nucl. Fusion, 49(5):055009, 2009. 
[12] P. Piovesan, M. Zuin, A. Alfier, D. Bonfiglio, F. Bonomo, A. Canton, S. Cappello, L. Carraro, R. Cavazzana, D.F. Escande, et al. Magnetic order and confinement improvement in highcurrent regimes of RFX-mod with MHD feedback control. Nucl. Fusion, 49(8):085036, 2009.

[13] M.E. Puiatti, M. Valisa, M. Agostini, F. Auriemma, F. Bonomo, L. Carraro, A. Fassina, M. Gobbin, R. Lorenzini, B. Momo, et al. Internal and external electron transport barriers in the RFX-mod reversed field pinch. Nucl. Fusion, 51(7):073038, 2011.

[14] J.P. Dahlburg, D.C. Montgomery, G.D. Doolen, and L. Turner. Driven, steady-state RFP computations. J. Plasma Phys., 40(01):39-68, 1988.

[15] A.Y. Aydemir, D.C. Barnes, E.J. Caramana, A.A. Mirin, R.A. Nebel, D.D. Schnack, and A.G. Sgro. Compressibility as a feature of field reversal maintenance in the reversed-field pinch. Phys. Fluids, 28:898, 1985.

[16] S. Cappello and D. Biskamp. Reconnection processes and scaling laws in reversed field pinch magnetohydrodynamics. Nucl. Fusion, 36(5):571, 1996.

[17] X. Shan, D.C. Montgomery, and H. Chen. Nonlinear magnetohydrodynamics by Galerkin-method computation. Phys. Rev. A, 44(10):6800-6818, 1991.

[18] X. Shan and D.C. Montgomery. On the role of the Hartmann number in magnetohydrodynamic activity. Plasma Phys. Controlled Fusion, 35(5):619, 1993.

[19] X. Shan and D.C. Montgomery. Global searches of Hartmann-number-dependent stability boundaries. Plasma Phys. Controlled Fusion, 35:1019, 1993.

[20] S. Cappello and D.F. Escande. Bifurcation in viscoresistive MHD: The Hartmann number and the reversed field pinch. Phys. Rev. Lett., 85(18):3838, 2000.

[21] M. Onofri, F. Malara, and P. Veltri. Compressibility effects in the dynamics of the reversed-field pinch. Phys. Rev. Lett., 101(25):255002, 2008.

[22] M. Onofri, F. Malara, and P. Veltri. Effects of compressibility and heating in magnetohydrodynamics simulations of a reversed field pinch. Phys. of Plasmas, 16:052508, 2009.

[23] D. Bonfiglio, S. Cappello, and D.F. Escande. Dominant electrostatic nature of the reversed field pinch dynamo. Phys. Rev. Lett., 94(14):145001, 2005.

[24] S. Cappello, D. Bonfiglio, D.F. Escande, S.C. Guo, I. Predebon, F. Sattin, M. Veranda, P. Zanca, C. Angioni, L. Chacon, et al. Equilibrium and transport for quasi-helical reversed field pinches. Nucl. Fusion, 51(10):103012, 2011.

[25] D. Bonfiglio, M. Veranda, S. Cappello, D.F. Escande, and L. Chacón. Experimental-like helical self-organization in reversed-field pinch modeling. Phys. Rev. Lett., 111(8):085002, 2013.

[26] D Terranova, D Bonfiglio, A H Boozer, A W Cooper, M Gobbin, S P Hirshman, R Lorenzini, L Marrelli, E Martines, B Momo, N Pomphrey, I Predebon, R Sanchez, G Spizzo, M Agostini, A Alfier, L Apolloni, F Auriemma, M Baruzzo, T Bolzonella, F Bonomo, M Brombin, A Canton, S Cappello, L Carraro, R Cavazzana, S Dal Bello, R Delogu, G De Masi, M Drevlak, A Fassina, A Ferro, P Franz, E Gaio, E Gazza, L Giudicotti, L Grando, S C Guo, P Innocente, D LpezBruna, G Manduchi, G Marchiori, P Martin, S Martini, S Menmuir, S Munaretto, L Novello, R Paccagnella, R Pasqualotto, G V Pereverzev, R Piovan, P Piovesan, L Piron, M E Puiatti, M Recchia, F Sattin, P Scarin, G Serianni, A Soppelsa, S Spagnolo, M Spolaore, C Taliercio, M Valisa, N Vianello, Z Wang, A Zamengo, B Zaniol, L Zanotto, P Zanca, and M Zuin. A 3d approach to equilibrium, stability and transport studies in rfx-mod improved regimes. Plasma Physics and Controlled Fusion, 52:124023, 2010.

[27] D. Bonfiglio, L. Chacón, and S. Cappello. Nonlinear three-dimensional verification of the SPECYL and PIXIE3D magnetohydrodynamics codes for fusion plasmas. Phys. of Plasmas, 17:082501, 2010.

[28] N. Mizuguchi, A. Sanpei, S. Fujita, K. Oki., H. Himura., S. Masamune, and K. Ichiguchi. Modeling of formation of helical structures in reversed-field pinch. Plasmas Fusion Res., 7:2403117, 2012.

[29] D.C. Montgomery and X. Shan. Toroidal resistive MHD equilibria. Comments Plasma Phys. Controlled Fusion, 15:315, 1994.

[30] L.P.J. Kamp and D.C. Montgomery. Toroidal steady states in visco-resistive magnetohydrody- 
namics. J. Plasma Phys., 70(2):113-142, 2004.

[31] D.C. Montgomery, J.W. Bates, and R.H. Lewis. Resistive magnetohydrodynamic equilibria in a torus. Phys. of Plasmas, 4(4):1080, 1997.

[32] J.A. Morales, W.J.T. Bos, K. Schneider, and D. Montgomery. Magnetohydrodynamically generated velocities in confined plasma. http://hal.archives-ouvertes.fr/hal-00849742, 2013.

[33] J.A. Morales, M. Leroy, W.J.T. Bos, and K. Schneider. Simulation of confined magnetohydrodynamic flows with dirichlet boundary conditions using a pseudo-spectral method with volume penalization. J. Comp. Phys., 274:64, 2014.

[34] S. Cappello. Bifurcation in the MHD behaviour of a self-organizing system: the reversed field pinch (RFP). Plasma Phys. Controlled Fusion, 46(12B):B313, 2004.

[35] R. Ikezoe, K. Oki, T. Onchi, Y. Konishi, M. Sugihara, S. Fujita, A. Sanpei, H. Himura, and S. Masamune. Extended operational regimes and MHD behavior in a low-aspect-ratio reversed field pinch in RELAX. Plasma Phys. Controlled Fusion, 53(2):025003, 2011.

[36] J.W. Bates and D.C. Montgomery. Toroidal visco-resistive magnetohydrodynamic steady states contain vortices. Phys. of Plasmas, 5:2649, 1998.

[37] J.A. Morales, W.J.T. Bos, K. Schneider, and D.C. Montgomery. Intrinsic rotation of toroidally confined magnetohydrodynamics. Phys. Rev. Lett., 109:175002, 2012.

[38] A.Y. Aydemir. An intrinsic source of radial electric field and edge flows in tokamaks. Nucl. Fusion, 49(6):065001, 2009.

[39] S. Pamela, G. Huysmans, and S. Benkadda. Influence of poloidal equilibrium rotation in mhd simulations of edge-localized modes. Plasma Physics and Controlled Fusion, 52(7):075006, 2010.

[40] G. R. Dennis, S. R. Hudson, D. Terranova, P. Franz, R. L. Dewar, and M. J. Hole. Minimally constrained model of self-organized helical states in reversed-field pinches. Phys. Rev. Lett., 111:055003, 2013.

[41] J.B. Taylor. Relaxation and magnetic reconnection in plasmas. Reviews of Modern Phys., 58(3):741, 1986.

[42] H. Toyama, N. Asakura, K. Hattori, N. Inoue, S. Ishida, S. Matsuzuka, K. Miyamoto, J. Morikawa, Y. Nagayama, H. Nihei, S. Shinohara, Y. Ueda, K. Yamagishi, and Z. Yoshida. Unknown. In Proc. 12th Europ. Conf. Controlled Fusion Plasma Phys., Budapest, volume I, page 602. L. Pocs and A. Montvai (European Physical Society, Geneva), 1985.

[43] J.N. DiMarco. The ZT-40M experiment. Proc. of the International School of Plasma Physics, Course on Mirx_r-Based and Field-Reversed Approaches to Magnetic Fusion, Villa Monastero, Varenna, Italy, 2, 1983.

[44] J.B. Taylor. Relaxation of toroidal plasma and generation of reverse magnetic fields. Phys. Rev. Lett., 33(19):1139, 1974.

[45] S. Cappello, D. Bonfiglio, D.F. Escande, S.C. Guo, A. Alfier, R. Lorenzini, and RFX Team. The reversed field pinch toward magnetic order: a genuine self-organization. In AIP Conference Proceedings, volume 1069, page 27, 2008. 